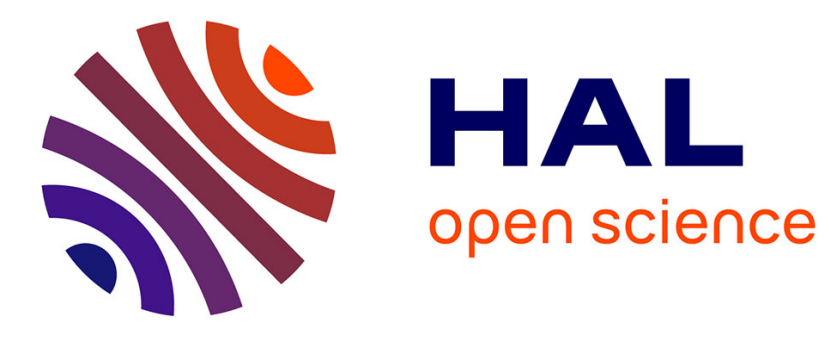

\title{
Gender and 3D Facial Symmetry: What's the Relationship?
}

Baiqiang Xia, Boulbaba Ben Amor, Hassen Drira, Mohamed Daoudi, Lahoucine Ballihi

\section{- To cite this version:}

Baiqiang Xia, Boulbaba Ben Amor, Hassen Drira, Mohamed Daoudi, Lahoucine Ballihi. Gender and 3D Facial Symmetry: What's the Relationship?. 10th IEEE Conference on Automatic Face and Gesture Recognition (FG 2013), Apr 2013, shanghai, China. hal-00771988

\section{HAL Id: hal-00771988 https://hal.science/hal-00771988}

Submitted on 9 Jan 2013

HAL is a multi-disciplinary open access archive for the deposit and dissemination of scientific research documents, whether they are published or not. The documents may come from teaching and research institutions in France or abroad, or from public or private research centers.
L'archive ouverte pluridisciplinaire HAL, est destinée au dépôt et à la diffusion de documents scientifiques de niveau recherche, publiés ou non, émanant des établissements d'enseignement et de recherche français ou étrangers, des laboratoires publics ou privés. 


\title{
Gender and 3D Facial Symmetry: What's the Relationship?
}

\author{
Baiqiang Xia*§ , Boulbaba Ben Amor ${ }^{\ddagger \S}$, Hassen Drira ${ }^{\ddagger \S}$, Mohamed Daoudi ${ }^{\ddagger}$ and Lahoucine Ballihi* ${ }^{\star}$ \\ * University Lille1 - Sciences and Technology, France. \\ $\ddagger$ Institut Mines-Télécom/TELECOM Lille1, France. \\ $\S$ LIFL (UMR 8022 Lille 1/CNRS), France.
}

\begin{abstract}
Although it is valuable information that human faces are approximately symmetric, in the literature of facial attributes recognition, little consideration has been given to the relationship between gender, age, ethnicity, etc. and facial asymmetry. In this paper we present a new approach based on bilateral facial asymmetry for gender classification. For that purpose, we propose to first capture the facial asymmetry by using Deformation Scalar Field (DSF) applied on each 3D face, then train such representations (DSFs) with several classifiers, including Random Forest, Adaboost and SVM after PCAbased feature space transformation. Experiments conducted on FRGCv2 dataset showed that a significant relationship exists between gender and facial symmetry when achieving a $90.99 \%$ correct classification rate for the 466 earliest scans of subjects (mainly neutral) and $88.12 \%$ on the whole FRGCv2 dataset (including facial expressions).
\end{abstract}

\section{INTRODUCTION}

The human face presents a clear sexual dimorphism and face gender recognition is an extremely reliable and fast cognitive process [1]. People are remarkably accurate at deciding whether faces are male or female, even when cues from hair style, makeup, and facial hair are minimized [18]. In perception and understanding of computer vision domain colleagues [12], automatic gender classification by face is an active researching area. It could be used in human computer interaction (intelligent user interface, video games, etc.), visual surveillance, collecting demographic statistics for marketing (audience or consumer proportion analysis, etc.), security industry (access control, etc.) and psychology. Research on automatic gender classification by face goes back to the beginning of the 1990s. Since then, significant progress has been reported in the literature. Fundamentally, proposed techniques differ in (i) face images-2D or 3D (ii) choice of facial representation, ranging from simple raw 2D/3D pixels to complex features and (iii) design of classifiers, such as Neural Networks, SVM and boosting methods [2].

\section{A. Related work with 3D face images}

In [3], Liu et al. looked into the relationship between facial asymmetry and gender. They imposed a 2D grid on each 3D face mesh to represent the face with $3 \mathrm{D}$ grid points. With the selected symmetry plane which equally separates the face into right and left halves, the distance difference (Euclidean distances to the origin of the cylindrical coordinate system) between each point and its corresponding reflected point was calculated as height differences (HD), and the angle difference between their normal vectors was calculated as orientation differences (OD). Thus for each face, a HD-face and a OD-face were generated and presented in matrices. The relationships between gender and overall feature asymmetry were examined by comparing overall the mean value in both HD-face and OD-face. Results on 111 full 3D neutral face models of 111 subjects showed that statistically significant difference could be observed between genders with the overall OD facial asymmetry measurement. This result confirms early claims in anthropometrical study, which claims that male faces generally possess a larger amount of asymmetry than female ones [13], [16]. They also defined a local symmetry measurement named Variance Ratio (VR). With a VR threshold, discriminative lowdimensional subspaces of HD- and OD-face were generated by LDA, and then fed to a linear classifier to investigate the relationship between gender and local feature asymmetry. With HD-face and on OD-face, they achieved $91.16 \%$ and $96.22 \%$ gender recognition rate in testing, respectively. The range and intensity modalities of the face provide different cues of demographic information. In [4], Lu et al. provided an integration scheme for range and intensity modalities to classify ethnicity (Asian and Non-Asian) and gender (Male and Female). SVM was used to extract posterior probabilities from normalized range and intensity images. Posterior probability values of range and intensity were then fused with equal weight and compared directly for ethnicity and gender classification. A mixture of two frontal 3D face databases (UND and MSU databases) was used for experiments. The best gender classification result using 10 -fold cross-validation reported was $91 \%$. Note that for males the result was $95.6 \%$ and for females the result was $83 \%$. That is probably due to there are unequal numbers of scans in the dataset between genders. Statistically there are differences in geometry facial features between different genders, such as in the hairline, forehead, eyebrows, eyes, cheeks, nose, mouth, chin, jaw, neck, skin and beard regions [8]. In [6], Han et al. presented a geometry feature based approach for 3D-face gender classification. The volume and area of the forehead, and their corresponding ratio to nose, eyebrows, cheeks and lips were defined to generate feature vectors. RBF-SVM was then applied to classify gender. They selected 61 frontal 3D face meshes from the GavabDB database, and carried out 5 experiments, with each experiment containing 48 faces for training and 13 for testing. The average correctness reported was $82.56 \%$. In [5], Wu et al. used 2.5D facial surface normals (needle-maps) recovered with Shape From Shading (SFS) from intensity images for gender classification. The recovered needle-maps presented in PGA (Principle Geodesic Analysis) parameters not only contain facial shape information, but also the illumination intensity 
implicitly. Training feature vectors were extracted by LDA from these needle-maps and then used in constructing Gaussian models to classify gender. They selected 260 2D frontal face images from the UND Database. Experiments were done 10 times with 200 faces randomly selected for training and the remaining 60 faces were selected for testing. The best average gender recognition rate reported was $93.6 \%$ with both shape and texture considered. In [7], $\mathrm{Hu}$ et al. proposed a fusionbased gender classification method for 3D frontal faces. Each 3D face shape was separated into four face regions using face landmarks. With the feature of each region, classifications were done using SVM on the UND and another database captured by themselves. Results showed that the upper region of the face contains the highest amount of discriminative gender information. Fusion was applied to the results of four face regions and the best result reported was $94.3 \%$.

Recently, in [12], Toderici et al. employed MDS (MultiDimensional Scaling) and wavelets on 3D face meshes for gender classification. They took 1121 scans of Asian subjects and 2554 scans of White subjects in FRGCv2 for ethnicity and gender classification. Experiments were carried out subjectindependently with no common subject used in the tesing stage of 10-fold cross validation. With polynomial kernel SVM, they achieved about $93 \%$ correct gender classification rate with an unsupervised MDS approach, and about $94 \%$ correctness with the wavelets-based approach. Both approaches significantly outperformed the $\mathrm{kNN}$ and kernel-kNN approaches. More recently, in [17], Ballihi et al. extracted geometrical features (26 level curves and 40 radial curves) from 3D faces for gender classification. To form a high performance classifier with a minimal set of features, the Adaboost algorithm was used to select salient geometrical facial features. With the salient curves trained by 20 previous 3D faces in the FRGCv1 dataset, they obtained a correctness rate of $84.12 \%$ with the nearest neighbour algorithm when using the 466 earliest scans of the FRGCv2 dataset as the testing set. Note that, here, training and testing were done separately on different datasets. They also performed standard 10-fold cross-validation for the 466 earliest scans of FRGCv2, and obtained $86.05 \%$ with Adaboost.

\section{B. Methodology and contribution}

From the analysis above, it emerges that a large part of existing works on 2D- and 3D-based gender classification are based on local or global descriptions extraction followed by classification methods. To the best of our knowledge, only the study in [3] investigated the relationship between face symmetry and gender. It extracted measures of height differences (HD), and orientation differences (OD) on a defined grid of full 3D face models, instead of 2.5 face models. In addition, their process required manual landmarking of seven key-points on the face model. These constraints limit the application of their approach in non-cooperative contexts. In comparison, the main contribution of this work is providing a fully-automatic framework for gender classification, which explores the relationship between gender and 3D face symmetry with dense Deformation Scalar Field (DSF) defined on radial curves that are abstracted directly from each 3D face. The DSFs grounding on Riemannian shape analysis are capable to densely capture the shape differences in 3D faces (such as bilateral 3D facial asymmetry) through face representation of radial curves. An overview of the proposed approach is depicted in Fig. 1. Firstly, during preprocessing, together with nose tip and symmetry plane detection, hole-filling, cropping and smoothing are applied to each scan. The preprocessed face surface is noted with $\boldsymbol{S}$. Secondly, $\boldsymbol{S}$ is approximated by collections of radial curves emanating from the nose tip after ICP alignment. A dense Deformation Scalar Field (DSF) is then computed on each face to capture, pair-wisely, the shape differences between corresponding symmetrical radial curves on each indexed point. Thirdly, the obtained dense (high-dimensional) DSFs are transformed using unsupervised Principal Component Analysis to obtain a low-dimensional feature space. Finally, standard 10-fold cross-validations using well-known Machine Learning techniques, including Random Forest, Boosting and Support Vector Machine, are performed for gender classification, where each experiment contains two separate stages of training and testing.

The rest of the paper is organized as follows: in section II, we present our methodology for abstracting feature vectors containing 3D facial symmetry information; in section III, we detail the method for dimensional reduction of feature space and classifiers for gender classification; experimental results and the discussion are presented in section IV while section $\mathrm{V}$ makes the conclusion.

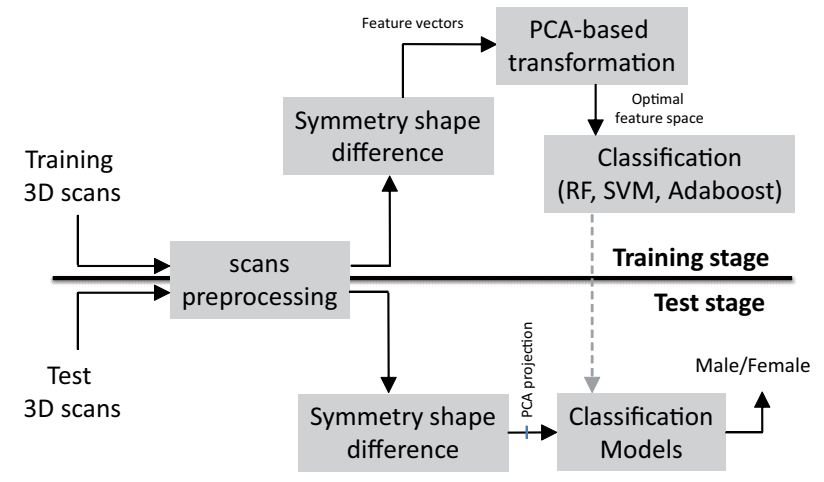

Fig. 1. Overview of the proposed approach.

\section{LOCAL ASYMMETRY MEASUREMENT}

The idea to capture local face asymmetry is to represent facial surface $S$ by a set of parameterized radial curves emanating from the nose tip. Such an approximation of facial surface can be seen as a solution to facial surface parameterization which captures the local shape information. Then, a Scalar Deformation Field (DSF), based on pair-wise shape comparison of corresponding symmetrical curves, is computed along these radial curves on each point. A similar framework has been used in [14] for 4D face expression recognition. More formally, a parametrized curve on the face, $\beta: I \rightarrow \mathbb{R}^{3}$, where $I=[0,1]$, is represented mathematically using the square-root velocity function [11], denoted by $q(t)$, according to: $q(t)=\frac{\dot{\beta}(t)}{\sqrt{\|\dot{\beta}(t)\|}}$. This specific parameterization has the advantage of capturing the shape of the curve and providing simple calculus. Let us define the space of such functions: $\mathcal{C}=\left\{q: I \rightarrow \mathbb{R}^{3},\|q\|=1\right\} \subset \mathbb{L}^{2}\left(I, \mathbb{R}^{3}\right)$, where $\|\cdot\|$ implies the $\mathbb{L}^{2}$ norm. With the $\mathbb{L}^{2}$ metric on its tangent spaces, $\mathcal{C}$ becomes a Riemannian manifold. Given two curves $q_{1}$ and $q_{2}$, let $\psi$ denote a path on the manifold $\mathcal{C}$ between $q_{1}$ and $q_{2}$, 
$\dot{\psi} \in T_{\psi}(\mathcal{C})$ is a tangent vector field on the path $\psi \in \mathcal{C}$. In our case, as the elements of $\mathcal{C}$ have a unit $\mathbb{L}^{2}$ norm, $\mathcal{C}$ is a Hypersphere in the Hilbert space $\mathbb{L}^{2}\left(I, \mathbb{R}^{3}\right)$. The geodesic path $\psi^{*}$ between any two points $q_{1}, q_{2} \in \mathcal{C}$ is simply given by the minor arc of great circle connecting them on this Hypersphere, $\psi^{*}:[0,1] \rightarrow \mathcal{C}$, given by $(1):$

$$
\psi^{*}(\tau)=\frac{1}{\sin (\theta)}\left(\sin ((1-\tau) \theta) q_{1}+\sin (\theta \tau) q_{2}\right)
$$

and $\theta=d_{\mathcal{C}}\left(q_{1}, q_{2}\right)=\cos ^{-1}\left(\left\langle q_{1}, q_{2}\right\rangle\right)$. We point out that $\sin (\theta)=0$ if the distance between the two curves is null, in other words $q_{1}=q_{2}$. In this case, for each $\tau, \psi^{*}(\tau)=q_{1}=q_{2}$. The tangent vector field on this geodesic $\dot{\psi}^{*}:[0,1] \rightarrow T_{\psi}(\mathcal{C})$ is then given by (2):

$$
\dot{\psi}^{*}=\frac{d \psi^{*}}{d \tau}=\frac{-\theta}{\sin (\theta)}\left(\cos ((1-\tau) \theta) q_{1}-\cos (\theta \tau) q_{2}\right)
$$

Knowing that on a geodesic path, the covariant derivative of its tangent vector field is equal to $0, \dot{\psi}^{*}$ is parallel along the geodesic $\psi^{*}$ and we shall represent it with $\left.\dot{\psi}^{*}\right|_{\tau=0}$. Accordingly, (2) becomes:

$$
\left.\dot{\psi}^{*}\right|_{\tau=0}=\frac{\theta}{\sin (\theta)}\left(q_{2}-\cos (\theta) q_{1}\right)
$$

with $\theta \neq 0$. Thus, $\left.\dot{\psi}^{*}\right|_{\tau=0}$ is sufficient to represent this vector field; the remaining vectors can be obtained by parallel transport of $\left.\dot{\psi}^{*}\right|_{\tau=0}$ along the geodesic $\psi^{*}$. In practice, the first step to capture the bilateral symmetry difference on the 3D face $S$ is to extract the radial curves. Let $\beta_{\alpha}$ denotes the radial curve that makes an angle $\alpha$ with the symmetry plane $P\left(t, \overrightarrow{n_{h}}\right)$ from the frontal view of $\boldsymbol{S}$, and $\beta_{2 \pi-\alpha}$ denotes the corresponding symmetrical curve that makes an angle $2 \pi-\alpha$ with $P\left(t, \overrightarrow{n_{h}}\right)$. The tangent vector field $\dot{\psi}_{\alpha}{ }^{*}$ that represents the energy needed to deform $\beta_{\alpha}$ to $\beta_{2 \pi_{*} \alpha}$ is then calculated. We consider only the magnitude of $\dot{\psi}_{\alpha}{ }^{*}$ at each point, located in curve $\beta_{\alpha}$ with index $k$, to build a Deformation Scalar Field on the facial surface, $V_{\alpha}^{k}=\left.|| \dot{\psi}_{\alpha}^{*}\right|_{(\tau=0)}(k) \|$, where $\alpha$ denotes the angle with $P\left(t, \overrightarrow{n_{h}}\right)$ from the frontal view and $k$ denotes the index of point on this curve. This Deformation Scalar Field quantifies the deformation between corresponding symmetrical curves on each point.

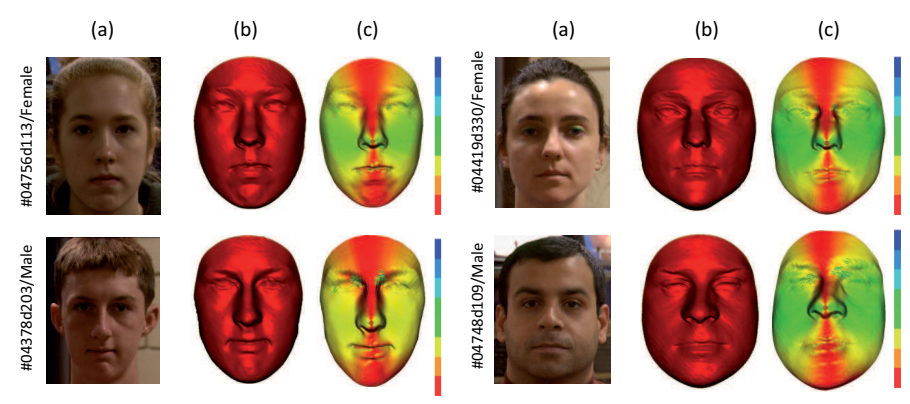

Fig. 2. Illustrations of DSF on face. (a) 2D intensity image; (b) preprocessed 3D face surface $S$; (c) colour-map of the DSF mapped on $S$
Some examples illustrating this calculus are shown in Fig. 2. For each subject, the face in column (a) shows the 2D intensity image; column (b) illustrates the preprocessed 3D face surface $S$; column (c) shows the symmetry degree as a colormap of the Deformation Scalar Field mapped on $S$. The color bars are shown on the right. The warm color means the minimum deformation and cold color signifies the maximum deformation. The hotter the color, the lower magnitude of the bilateral asymmetry. In our work, the DSFs are generated with 200 radial curves extracted from each face and 100 indexed points on each curve. Thus, the total volume is less than 50 VR values computed on subregions of HD or OD face, our experiment with DSF descriptor outperfoms significantly in density. The average time consumed for extracting all 200 curves for each face is 1.048 second, and for generating the bilateral symmetry descriptor (DSF) on all $200 \times 100$ points of each face is 0.058 second. Thus the total computation time (including preprocessing) for each scan is less than 1.2 second.

\section{GENDER CLASSIFICATION}

Face-based gender classification is a binary problem which classifies the gender of query face to male or female. Since the volume of each DSF is as many as 20000, for gender classification, we first perform PCA on the DSFs to generate a low dimensional feature space. PCA is a mathematical procedure using orthogonal transformation to convert a set of observations of variables into a set of linearly independent variables (principal components). Dimensionality reduction is achieved by selecting a number of principal components as PCA output. PCA could work in its natural way without supervision. We then carry out experiments with three wellknown machine learning algorithms, namely Random Forest, Adaboost and SVM. Random Forest is an ensemble learning method that grows a forest of classification trees based on random selected feature and threshold. To classify an object, the input vector is fed to each tree and each tree gives a classification result. The forest selects the result by simple majority voting. It is reported that face classification by Random Forest achieves lower error rate than some popular classifiers, including SVM [9]. However, as far as we know, there is no reported work in the literature of face-based gender classification using Random Forest. The idea of SVM is that samples of different classes can be separated in high dimensional space using transformed features. The transformation is generated by kernel functions, where RBF and polynomial kernels are popular ones [1]. In gender classification literature, many works have been done with SVM and it has demonstrated good performance compared to other classifiers [1], [6]. In the Adaboost algorithm, specific features are selected. The weak classifiers that used with the selected features then form together a stronger classifier. The features and weak classifiers can be anything as long as they classify given data examples to specified classes [1]. Comparison made in [10] shows Adaboost could perform even better than representative SVM.

\section{EXPERIMENTS AND DISCUSSIONS}

The FRGCv2 database was collected by researchers of the University of Notre Dame and contains 4007 3D face scans of 466 subjects with differences in gender, ethnicity, age and expression [15]. For gender, there are 1848 scans of 203 female 
subjects and 2159 scans of 265 male subjects. The ages of subjects range from 18 to 70 years old, with $92.5 \%$ in the domain of 18-30 years old. When considering ethnicity, there are 2554 scans of 319 White subjects, 1121 scans of 99 Asian subjects, 78 scans of 12 Asian-southern subjects, 16 scans of 1 Asian and Middle-east subject, 28 scans of 6 Black-or-African American subjects, 113 scans of 13 Hispanic subjects, and 97 scans of 16 ethnicity-Unknown subjects. About $60 \%$ of the faces have neutral expression, and the others show expressions of disgust, happiness, sadness and surprise. All the scans in FRGCv2 are near-frontal. With FRGCv2, we performed two types of experiments with different data selection. The first type is designed mainly for examining the robustness of our approach to variations of age and ethnicity in faces. It uses the 466 earliest scans of each subject in FRGCv2, in which more than $93 \%$ of scans are neutral-frontal. The second type extents to examine the robustness of our approach to variations of expressions. It enrolls all the 4007 scans in FRGCv2, about $40 \%$ of which are expressive faces, in a subject-independent fashion. For both types of experiments, results are generated with standard 10 -fold cross-validation.

\section{A. Robustness to variations of age and ethnicity}

Among the 466 earliest scans, there are 431 neutralfrontal scans and 35 expressional-frontal scans. In 10-fold cross validation, the 466 scans are randomly partitioned into 10 folds with each fold containing 46-47 scans. Each round 9 of the 10 folds are used for training while the remaining fold is used for testing. We perfomed experiments with all the three classifiers seperately. The average recognition rate and standard deviation for 10 rounds then give a statistical significant performance measure of proposed methodology. The relationship between the performance of classifiers and the number of PCA-based feature vectors is shown in Fig. 3. It evidently demonstrates significant relationship exists between gender and facial symmetry, which echos previous findings in anthropometrical study [13]. We could also see the results of different classifiers are very close to each other, and the results of each classifier change little when the number of feature vectors changes. That means our approach has comparable performace with changes of classifiers and number of PCAbased feature vectors. We also obtained gender classification results for Random Forest with different number of trees in Fig. 4. It shows again that close relationship exists between face symmetry and gender. The best average gender recognition rate is $90.99 \%$ with standard deviation of 5.99 , achieved by Random Forest with 39 trees and 20 PCA-based feature vectors. The results are detailed in confusion matrix in Table 1. Note that the recognition rate for females $(89.66 \%)$ is slightly lower than the males $(92.02 \%)$. This difference is probably due to more male faces were used for training. We also performed a 10-fold 100-repetition experiment with Random Forest under the same setting, which resulted at $88.12 \%$ average correctness with standard deviation of $5.53 \%$.

TABLE I. CONFUSION MATRIX USING RANDOM FOREST

\begin{tabular}{|c|c|c|}
\hline$\%$ & Female & Male \\
\hline Female & 89.66 & 10.34 \\
\hline Male & 7.98 & 92.02 \\
\hline \multicolumn{2}{|l|}{ Average recognition rate $=90.99 \pm 5.99 \%$} \\
\hline
\end{tabular}

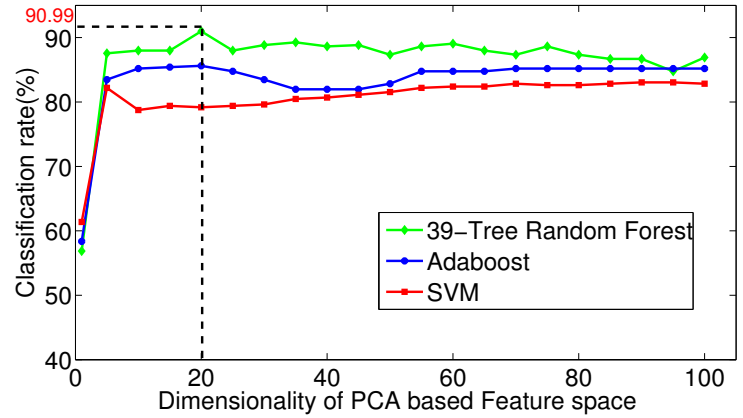

Fig. 3. Results of different classifiers with different number of PCA-based feature vectors on 466 earliest scans

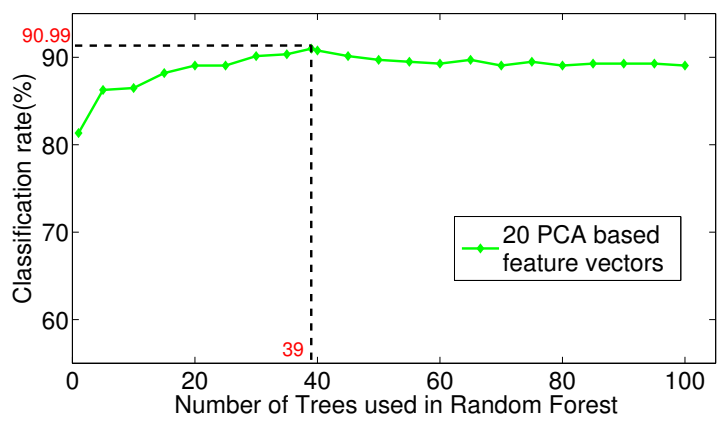

Fig. 4. Results of Random Forest using different number of trees on 466 earliest scans

Fig. 5 illustrates colormaps of DSF on female faces with difference in age and Fig. 6 illustrates colormaps of DSF on male faces with difference in ethnicity. The informations of age, ethnicity and id of scans are presented in 2D images in the upper row of each figure. With Fig. 5 and Fig. 6, we could observe that deformations in scans of both gender convey visually symmetrical pattern, where the colormap of left-face is globly in symmetry with the right-face although sutble local asymmetry exists. Low-level deformations (red color) uaually locate near the symmery plane and high-level deformations (yellow and green colors) happen more frequently in farther areas. These globle observations do not change significantly with changes of age and ethnicity. At the same time, we could perceive that the deformations in female faces changes obviously more smoothly than males. Without proven, we believe that these common globle patterns contribute to the robustness of our approach to variations of age and ethnicity to some extend.

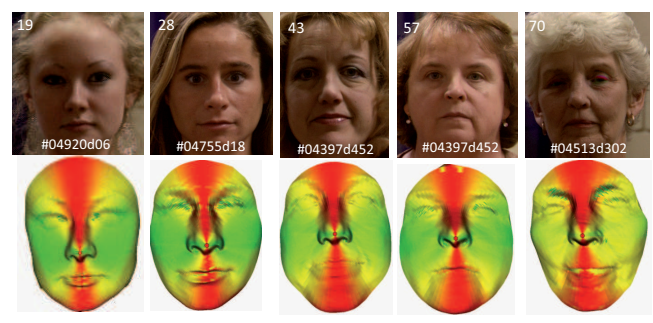

Fig. 5. Illustration of DSF on face with different Age. 


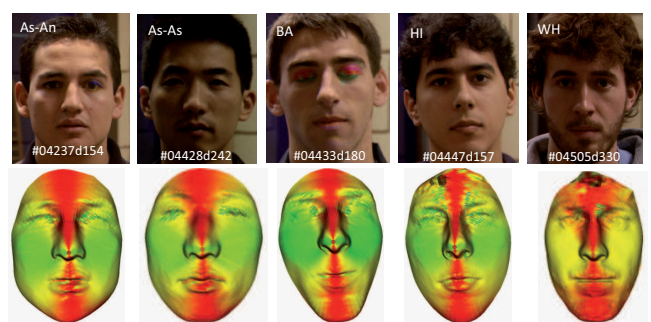

Fig. 6. Illustration of DSF on face with different Ethnicity

\section{B. Robustness to variation of expression}

With the results in previous section, we reasonably assume that Random Forest performs better than other classifiers in gender classification on this database. Thus we only use Random Forest for the experiments on the whole FRGCv2 dataset. After preprocessing, we obtained 4005 well preprocessed scans for extracting DSFs. The failed two scans (with scan id $04629 d 148$ and $04815 d 208$ ) were resulted from wrong nose tip detection. Considering that the ratio of failure is rathor tiny $(2 / 4007<0.0005)$, we simply omit the influence of the two failed scans for result generation. With the 4005 well preprocessed scans, we performed PCA to the DSFs after DSF extraction, and then 10-fold subject-independent crossvalidation with 50-Tree Random Forest. For each round, scans of 46 subjects are randomly selected for testing, with scans of remaining subjects dedicated for training. For all the 10 rounds of experiments, no common subjects are used in testing.

The relationship between classification result of Random Forest and number of PCA-based feature vectors is shown in Fig. 7. The best result achieved with 30 PCA-based feature vectors is $88.12 \% \pm 5.53$. Considering that FRGCv2 is a really big and challenging database which contains more than 4000 scans with various changes in age, ethnicity and expression, we could claim even more confidently that significant relationship exists between gender and 3D facial symmetry, and our method is effective and robust to age, ethnicity and even expressions in gender classification.

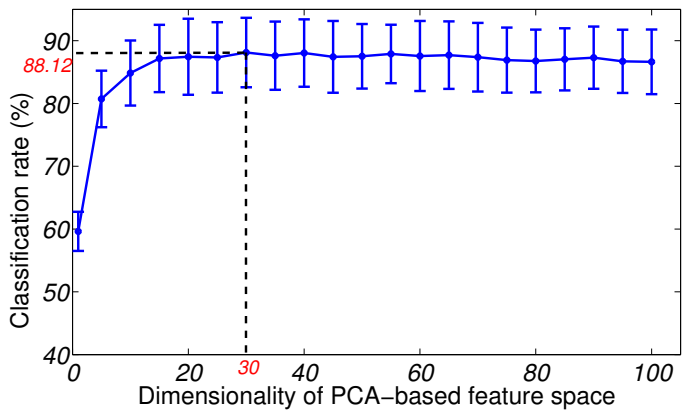

Fig. 7. Results of Random Forest with different number of PCA-based feature vectors on whole FRGCv2 dataset.

Fig. 8 shows colormaps of DSFs generated for a subject with different expressions. Similar with the perceptions in Fig. 5 and Fig. 6, we perceive again that the deformations on both sides of face are roughly in symmetry, although in some areas like eye corners and lips there are tiny local assymmetry. Lowlevel deformations (red color) always locate near the symmery plane and high-level deformations (yellow and green colors) happen more frequently in farther areas. All these visible patterns do not change significantly with expression variation. We believe these patterns contribute to the robustness of our approach to expression changes.
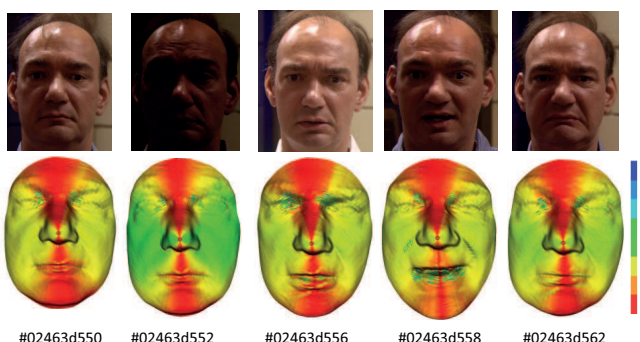

Fig. 8. Illustration of DSF on face with different expressions

\section{Comparison with state of the art}

Table 2 gives a comparison of previous works. With huge differences in dataset, landmarking, experiment settings and so on, it is difficult to compare and rank these works simply according to the result values. Compared with our work, works in [6], [5] , [3] are based on relatively smaller dataset which leave doubts of the statistical significance of their approaches on larger and more challenging datasets, works in [6], [5], [4], [3] require manual landmarking thus their approaches are not fully-automatic, works in [7], [6], [5], [3] use different experiment settings other than the most prevailing 10-fold crossvalidation. Our work addressed gender classification in a fully automatic way without manual landmarking, experimented on a large dataset FRGCv2 which contains challenging variations in expression, age and ethnicity, and reached comparable results with literature. The nearest works to ours are done by Ballihi et al. in [17] and George et al. With the 466 Earliest scans of FRGCv2 and standard 10-fold cross-validation, Ballihi et al. achieved $86.05 \%$ correctness in [17], while we achived a much higher result of $90.99 \%$ with Random Forest. In [12], George et al. also performed automatic 10-fold crossvalidation on FRGCv2 dataset in subject-independent fashion. Their results are based on 3676 scans of White and Asian subjects and relatively higher in value comparing with ours. However, our experiment on the whole FRGCv2 dataset has covered all the 4007 scans in FRGCv2, thus encountered more challenges from data amount and ethnicity variation. Additionally, during experiments we found an error in metadata of FRGCv2, which mislabled gender of a subject (with id 04662, female indeed) to male and resulted in 8 wrongly labeled scans. we corrected it and carried out our works with the correct meta-data.

\section{CONCLUSIONS}

In this paper, we have proposes a fully automatic approach based on 3D facial bilateral symmetry for gender classification. With facial shape represented by collections of radial curves, a Deformation Scalar Field is calculated on each indexed point of symmetrical curves in preprocessed face. PCA is then applied on the Deformation Scalar Fields to generate a low-dimensional feature space. Obtained feature vectors are then used as input to classifiers including Random Forest, 
TABLE II. COMPARISON OF OUR APPROACH TO EARLIER STUDIES.

\begin{tabular}{|c|c|c|c|c|c|c|c|}
\hline Author & Dataset & $\begin{array}{l}\text { Manual land- } \\
\text { marks }\end{array}$ & Features & Classifiers & Experiment settings & Results reported & Notes \\
\hline $\begin{array}{l}\text { Ballihi et al. } \\
\text { [17] }\end{array}$ & $\begin{array}{l}20 \text { previous faces } \\
\text { of FRGC-1.0 and } \\
466 \text { earliest scans } \\
\text { of FRGCv2 dataset }\end{array}$ & No & $\begin{array}{lr}20 \text { salient } & \text { curves } \\
\text { selected } & \text { by } \\
\text { Adaboost } & \text { with } \\
\text { faces of FRGC-1.0 }\end{array}$ & $\begin{array}{l}\text { Adaboost } \\
\text { (Classification } \\
\text { done only with } \\
\text { scans of FRGCv2) }\end{array}$ & $\begin{array}{l}\text { 10-fold } \quad \text { cross- } \\
\text { validation }\end{array}$ & $86.05 \%$ & $\begin{array}{l}\text { Using only } \\
\text { shape }\end{array}$ \\
\hline $\begin{array}{l}\text { Toderici et al. } \\
\text { [12] }\end{array}$ & $\begin{array}{l}3676 \text { scans from } \\
\text { FRGCv2 Dataset }\end{array}$ & No & Wavelets & $\begin{array}{l}\text { Polynomial kernel- } \\
\text { SVM }\end{array}$ & $\begin{array}{l}\text { l0-fold cross- } \\
\text { validation in subject- } \\
\text { independent fashion }\end{array}$ & $\begin{array}{l}\text { Male: } 94 \% \pm 5 \mathrm{Fe}- \\
\text { male: } 93 \% \pm 4\end{array}$ & $\begin{array}{l}\text { Using only } \\
\text { shape }\end{array}$ \\
\hline Hu et al. [7] & $\begin{array}{l}729 \text { frontal } \\
3 D \text { scans from } \\
\text { UND and } 216 \\
\text { neutral-frontal } 3 D \\
\text { scans captured } \\
\text { themselves }\end{array}$ & No & $\begin{array}{l}\text { Curvature-based } \\
\text { shape index for } \\
\text { five face regions }\end{array}$ & $R B F-S V M$ & $\begin{array}{ll}5 \text {-fold } & \text { cross- } \\
\text { validation } & \end{array}$ & $94.03 \%$ & $\begin{array}{l}\text { Using only } \\
\text { shape }\end{array}$ \\
\hline Han et al. [6] & $\begin{array}{l}61 \text { 2nd capture of } \\
61 \text { sujects in Gav- } \\
a b D B \text { Dataset }\end{array}$ & Yes & Geometry Features & $R B F-S V M$ & $\begin{array}{ll}\text { 5-fold } & \text { cross- } \\
\text { validation } & \end{array}$ & $82.56 \% \pm 0.92$ & $\begin{array}{l}\text { Using only } \\
\text { shape }\end{array}$ \\
\hline Wu et al. [5] & $\begin{array}{l}\text { Needle maps re- } \\
\text { covered from } 260 \\
2 D \text { images of } U N D \\
\text { dataset }\end{array}$ & Yes & PGA features & $\begin{array}{l}\text { Posteriori Proba- } \\
\text { bilities }\end{array}$ & $\begin{array}{l}6 \text { experiments with } \\
\text { each contains } 200 \\
\text { scans for training } \\
\text { and } 60 \text { for testing }\end{array}$ & $93.6 \% \pm 0.040$ & $\begin{array}{lr}\text { Using } & \text { both } \\
\text { shape } & \text { and } \\
\text { texture } & \end{array}$ \\
\hline Lu et al. [4] & $\begin{array}{l}1240 \text { scans of } 376 \\
\text { subjects from UND } \\
\text { and MSU datasets }\end{array}$ & Yes & $\begin{array}{l}\text { Concatenated Grid } \\
\text { element values }\end{array}$ & $\begin{array}{l}\text { SVM \& Posteriori } \\
\text { Probabilities }\end{array}$ & $\begin{array}{ll}10-\text { fold } & \text { cross- } \\
\text { validation }\end{array}$ & $91 \% \pm 0.03$ & $\begin{array}{lr}\text { Using } & \text { both } \\
\text { shape } & \text { and } \\
\text { texture } & \end{array}$ \\
\hline Liu et al. [3] & $\begin{array}{lr}11 \text { scans of } 111 \\
\text { subjects } & \text { from } \\
\text { University } & \text { of } \\
\text { South Florida } & \\
\end{array}$ & Yes & 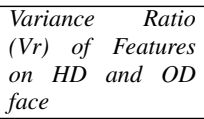 & $\begin{array}{l}\text { A linear classifier } \\
\text { develop themselves }\end{array}$ & $\begin{array}{l}100 \text { repetition with } \\
\text { half scans for training } \\
\text { and half for testing }\end{array}$ & $\begin{array}{l}H D: 91.16 \% \pm 3.15 \\
O D: 96.22 \% \pm 2.30\end{array}$ & $\begin{array}{l}\text { Using only } \\
\text { shape }\end{array}$ \\
\hline Our work $^{1}$ & $\begin{array}{l}466 \text { Earliest scans } \\
\text { of } F R G C v 2\end{array}$ & No & $D S F$ & Random Forest & $\begin{array}{l}\text { 10-fold } \\
\text { validation }\end{array}$ & $90.99 \% \pm 5.99$ & $\begin{array}{l}\text { Using only } \\
\text { shape }\end{array}$ \\
\hline Our work $^{2}$ & $\begin{array}{l}\text { All scans of } \\
F R G C v 2\end{array}$ & No & $D S F$ & Random Forest & $\begin{array}{l}\text { l0-fold cross- } \\
\text { validation in subject- } \\
\text { independent fashion }\end{array}$ & $88.12 \% \pm 5.53$ & $\begin{array}{l}\text { Using only } \\
\text { shape }\end{array}$ \\
\hline
\end{tabular}

Adaboost and SVM. Despite the wide range of age, ethnicity and expression of the faces in the selected dataset, we have achieved a gender classification result of $90.99 \% \pm 5.99$ with 466 earliest scans of subjects, and $88.18 \% \pm 5.53$ on the whole FRGCv2 dataset. In response to the question rised up in the title, we justify that significant relationship exists between gender and 3D facial symmetry with experiment results.

\section{ACKNOWLEDGMENTS}

This work is supported by the French research agency ANR through the 3D Face Analyzer project under the contract ANR 2010 INTB 030101 and partially supported by a Graduate Fellowship from NSFC to Baiqiang Xia.

\section{REFERENCES}

[1] A. Cellerino and D. Borghetti and F. Sartucci, "Sex differences in face gender recognition in humans", Brain Research Bulletin, vol. 63, 2004.

[2] J. Ylioinas and A. Hadid and M. Pietikainen, "Combining Contrast Information and Local Binary Patterns for Gender Classification", Image Analysis, vol. 6688, 2011, pp 676-686.

[3] Y. Liu and J. Palmer, "A quantified study of facial asymmetry in 3D faces", Analysis and Modeling of Faces and Gestures,2003, pp. 222229.

[4] X. Lu and H. Chen and A. Jain, "Multimodal facial gender and ethnicity identification", Proceedings of the 2006 international conference on Advances in Biometrics, 2006, pp. 554-561.

[5] J. Wu and W.A.P. Smith and E.R. Hancock, "Gender Classification using Shape from Shading",International Conference on Image Analysis and Recognition, 2007, pp. 499-508.

[6] X. Han and H. Ugail and I. Palmer, "Gender Classification Based on 3D Face Geometry Features Using SVM", CyberWorlds,2009, pp. 114-118.

[7] Y. Hu and J. Yan and P. Shi, "A fusion-based method for 3D facial gender classification", Computer and Automation Engineering (ICCAE), vol. 5 , 2010, pp. 369-372.
[8] "The main differences between male and female faces", www.virtualffs co.uk.

[9] A.Z. Kouzani and S. Nahavandi and K. Khoshmanesh, "Face classification by a random forest", TENCON 2007-2007 IEEE Region 10 Conference, 2007, pp 1-4.

[10] H. Lu and H. Lin, "Gender Recognition using Adaboosted Feature", Natural Computation, vol. 2, 2007, pp 646-650.

[11] A. Srivastava and E. Klassen and S.H. Joshi and I.H. Jermyn, "Shape Analysis of Elastic Curves in Euclidean Spaces", Pattern Analysis and Machine Intelligence, vol. 33, 2011, pp 1415 -1428.

[12] G. Toderici and S. O'Malley and G. Passalis and T. Theoharis and I. Kakadiaris, "Ethnicity- and Gender-based Subject Retrieval Using 3-D Face-Recognition Techniques", Int. J. Comput. Vision, vol. 89, 2011.

[13] LG. Farkas and G. Cheung, "Facial asymmetry in healthy North American Caucasians. An anthropometrical study", Angle Orthod,vol. 51, 1981, pp 70-7.

[14] H. Drira and B. Ben Amor and M. Daoudi and A. Srivastava and S. Berretti, "3D Dynamic Expression Recognition based on a Novel Deformation Vector Field and Random Forest", 21th Int. Conf. on Pattern Recognition, 2012.

[15] P.J. Phillips and P.J. Flynn and T. Scruggs and K.W. Bowyer and J. Chang and K. Hoffman and J. Marques and J. Min and W. Worek, "Overview of the face recognition grand challenge", Computer Vision and Pattern Recognition,vol. 1, 2005, pp 947 - 954.

[16] A. Little and B. Jones and C. Waitt and B. Tiddeman and D. Feinberg and D. Perrett and C. Apicella and F. Marlowe, "Symmetry Is Related to Sexual Dimorphism in Faces: Data Across Culture and Species", PLoS ONE,vol. 3, 2008.

[17] L. Ballihi and B. Ben Amor and M. Daoudi and A. Srivastava and D. Aboutajdine, "Boosting 3D-Geometric Features for Efficient Face Recognition and Gender Classification", IEEE Transactions on Information Forensics \& Security, vol. 7, 2012, pp 1766-1779.

[18] V. Bruce and AM. Burton and E. Hanna and P. Healey and O. Mason and A. Coombes and R. Fright and A. Linney, "Sex discrimination: how do we tell the difference between male and female faces?", Perception, vol. 22, 1993. 